\title{
REDUCED SYMMETRIC ALGEBRAS AND LINEAR SYZYGIES
}

\author{
MARK R. JOHNSON
}

1. Introduction. Let $M$ be a finitely generated module over a Noetherian domain $A$. A basic condition one would often like to know is the integrality of the symmetric algebra $S_{A}(M)$. A fundamental obstruction for this to hold is a restriction on the (local) number of generators: $\mu\left(M_{p}\right) \leq \operatorname{dim} A_{p}+\operatorname{rank} M-1$ for every non-minimal prime ideal $p$ of $A$. This condition is known as the $\mathcal{F}_{1}$ condition.

Unfortunately, the $\mathcal{F}_{1}$ condition alone is usually not enough to guarantee integrality; however, if the symmetric algebra happens to be equidimensional, then one may conclude (at least if $A$ has finite Krull dimension) that its associated spectrum is irreducible [9]. Thus, the integrality would follow if one also knew the reducedness of $S_{A}(M)$. It turns out that there are other situations where reducedness ensures the integrality.

Recently, Simis, Ulrich and Vasconcelos [7] have discovered one such case for the Zariski tangent algebra, which is the symmetric algebra $S_{A}\left(\Omega_{A / k}\right)$ of the module of differentials of an algebra $A$ essentially of finite type over a field $k$. For example, if $A$ is a local isolated singularity over the perfect field $k$, then the reducedness of the Zariski tangent algebra of $A$ implies its integrality-as long as $A$ is not defined by "too many" quadrics. They also establish a similar result for its normality, showing that it must in such a case coincide with its reflexive closure.

In this note, these results are extended to arbitrary modules, giving similar criteria for a reduced symmetric algebra to be integral, or a normal symmetric algebra to be its reflexive closure. The conditions translate into the module having sufficiently few "linear" relations. This also serves to clarify, and somewhat simplify, this part of the work of [7].

2010 AMS Mathematics subject classification. Primary 13A30.

Keywords and phrases. Symmetric algebra, linear syzygy, linear type.

Received by the editors on January 4, 2013, and in revised form on August 19, 2013. 
After establishing the reducedness result in Section 2 and treating normality in Section 3, we return to consider the reducedness in the case of ideals in Section 4, applying the result to the conormal module in a regular ambient ring, complementing the case of [7].

2. Reduced symmetric algebras. Let $(A, m, k)$ be a Noetherian local ring, and let $M$ be a finitely generated $A$-module. We will usually assume that $M$ is generically free, i.e., that $M_{p}$ is a free $A_{p}$-module for every $p \in \operatorname{Ass}(M)$. This condition holds if $M$ has a rank, for example if $A$ is a domain. We denote by minrank $M$ the smallest rank of $M$ locally at any minimal prime of $A$. By $\mu(M)$, we denote the minimal number of generators of $M$. By $\operatorname{edim} A$, we denote the embedding dimension of $A$, namely, $\mu(m)$. The embedding codimension of $A$, denoted by ecodim $A$, is $\operatorname{difference} \operatorname{edim} A-\operatorname{dim} A$. If $A \cong R / I$ is a quotient of a regular local ring $(R, m)$, and $I \subset m^{2}$, then ecodim $A=\operatorname{codim} I$.

We denote by $S(M)=S_{A}(M)$ the symmetric algebra of $M$. The Rees algebra $\mathcal{R}(M)$ is defined to be the quotient of $S(M)$ by its $A$ torsion. If $A$ is reduced (respectively integral) so is $\mathcal{R}(M)$. The analytic spread $\ell(M)$ is the Krull dimension of the fiber $\operatorname{ring} \mathcal{R}(M) \otimes_{R} k$. One has, in general, that $\ell(M) \leq \mu(M)$, where $\mu$ denotes the minimal number of generators. When $S(M)$ is $A$-torsionfree, or equivalently $\mathcal{R}(M)=S(M)$, we say that $M$ is of linear type. (Note that, when $A$ is integral, this simply means the integrality of $S(M)$.) In this case, $\mu(M)=\ell(M)$. (For more information concerning these notions, one may consult $[\mathbf{2}, \mathbf{8}, \mathbf{9}]$.)

We will also need the following notation.

Definition 2.1. Let $(A, m)$ be a Noetherian local ring, and let $M$ be a finitely generated $A$-module. Let

$$
A^{m} \stackrel{\phi}{\longrightarrow} A^{n} \longrightarrow M \longrightarrow 0
$$

be a minimal presentation of $M$. Let $\phi$ be an $n \times m$ matrix representation written as

$$
\phi=\left(\phi_{1} \mid \phi_{2}\right)
$$

where $\phi_{2}$ is a maximal submatrix having entries in $m^{2}$. We call $\phi_{1}$ the linear part of $\phi$ and the corresponding columns linear syzygies of $M$. 
We set

$$
\alpha_{1}(M)=\inf _{\phi}\left(\# \text { columns of } \phi_{1}\right),
$$

the infimum ranging over all minimal presentation matrices $\phi$ of $M$.

The definition of $\alpha_{1}(M)$ is given to make an invariant of $M$, independent of the choice of presentation: $\alpha_{1}(M)$ is the smallest number of columns, with entries not all in $\mathrm{m}^{2}$, that can appear in a minimal presentation matrix of $M$.

Note that we have $0 \leq \alpha_{1}(M) \leq \beta_{1}(M)$, where $\beta_{1}$ denotes the first Betti number.

Lemma 2.2. Let $(A, m)$ be a Noetherian local ring, and let $M$ be a finitely generated generically free $A$-module such that $\mu\left(M_{p}\right)=\ell\left(M_{p}\right)$ for every $p \neq m$, and $\mu(M) \neq \ell(M)$. If $S(M)$ satisfies $\left(R_{0}\right)$, then $\alpha_{1}(M) \geq \operatorname{edim} A$. Furthermore, if $\phi$ is a minimal presentation matrix of $M$, then $m=I_{1}\left(\phi_{1}\right)$.

Proof. Write $\phi=\left(\phi_{1} \mid \phi_{2}\right)$, so that $I_{1}\left(\phi_{2}\right) \subset m^{2}$. Set $T=$ $A\left[T_{1}, \ldots, T_{n}\right]$, let $L_{i}=\left(\left[T_{1}, \ldots, T_{n}\right] \phi_{i}\right)$ for $i=1,2$, and let $J=$ $\left(L_{1}, L_{2}\right)$, so $S(M) \cong T / J$. By $[3,3.4], m S(M)$ is a minimal prime of $S(M)$. Since $S(M)$ satisfies $\left(R_{0}\right)$, it follows that $m T_{m T}=J T_{m T} \subset$ $\left(L_{1}, m^{2}\right) T_{m T}$; hence, $m T_{m T}=L_{1} T_{m T}$ by Nakayama's lemma. Therefore,

$$
\begin{aligned}
r & =\# \text { columns of } \phi_{1} \geq \mu\left(L_{1}\right) \geq \mu\left(L_{1} T_{m T}\right) \\
& =\mu\left(m T_{m T}\right)=\mu(m)=\operatorname{edim} A .
\end{aligned}
$$

It follows that $\alpha_{1}(M) \geq \operatorname{edim} A$.

To verify the second statement, note that $L_{1} \subset I_{1}\left(\phi_{1}\right) T \subset m T$; hence, $m T_{m T}=I_{1}\left(\phi_{1}\right) T_{m T}$ as well. Since $I_{1}\left(\phi_{1}\right) \subset m$ are ideals of $A$, by flatness it follows that $m=I_{1}\left(\phi_{1}\right)$.

Theorem 2.3. Let $A$ be a reduced Noetherian ring, and let $M$ be a finitely generated generically free A-module satisfying

$$
\alpha_{1}\left(M_{p}\right)<\operatorname{edim}\left(A_{p}\right) \text { for every } p \in \operatorname{Spec}(A) \text { with } \ell\left(M_{p}\right)<\mu\left(M_{p}\right) \text {. }
$$

If $S(M)$ is reduced, then $S(M)$ is A-torsionfree. 
Proof. Since $S(M)$ is reduced, it suffices to show that the $A$-torsion of $S(M)$ is nilpotent. Equivalently, we show that $\mu\left(M_{p}\right)=\ell\left(M_{p}\right)$ for every prime $p([\mathbf{3}, 3.4])$. If $p \in \operatorname{Ass}(A)$, then this holds since $M$ is generically free. Supposing that the claim is false, we may localize at a minimal counterexample to assume that $(A, m)$ is local, that $\mu\left(M_{p}\right)=\ell\left(M_{p}\right)$ for every $p \neq m$, and that $\mu(M) \neq \ell(M)$. The hypothesis is now that $\alpha_{1}(M)<\operatorname{edim} A$. But this contradicts Lemma 2.2 and thus establishes the claim.

Corollary 2.4. Let $(A, m)$ be a reduced Noetherian local ring, and let $M$ be a finitely generated generically free A-module satisfying one of the following conditions:

(i) $M_{p}$ is of linear type for every $p \neq m$, and $M$ has a presentation matrix $\phi$ with $I_{1}(\phi) \subset m^{2}$;

(ii) $\beta_{1}\left(M_{p}\right)<\operatorname{edim}\left(A_{p}\right)$ for every non-minimal $p \in \operatorname{Spec}(A)$.

If $S(M)$ is reduced, then $S(M)$ is A-torsionfree.

Proof. In the first case, $\ell\left(M_{p}\right)=\mu\left(M_{p}\right)$ holds for every $p \neq m$, and $\alpha_{1}(M)=0$. For the second, one has that $\alpha_{1}(M) \leq \beta_{1}(M)$. Hence, the result follows from Theorem 2.3.

If $A$ is essentially of finite type over a field $k$, we may apply the result to the module of differentials $\Omega_{A / k}$, recovering results of [7].

Corollary 2.5. ([7,2.1]). Let $A$ be a reduced ring essentially of finite type over a perfect field $k$, and assume that, for every nonminimal $p \in \operatorname{Spec}(A), A_{p} \cong R / I$, where $(R, \mathfrak{n})$ is a regular local ring essentially of finite type over $k$, and $I \subset m^{2}$ is an $R$-ideal satisfying $\mu\left(I+\mathfrak{n}^{3} / \mathfrak{n}^{3}\right)<\operatorname{dim} R$.

If $S\left(\Omega_{A / k}\right)$ is reduced, then $S\left(\Omega_{A / k}\right)$ is A-torsionfree.

Proof. Using the Jacobian matrix of the local presentation, it follows that, for any non-minimal prime $p, \alpha_{1}\left(\left(\Omega_{A / k}\right)_{p}\right)=\alpha_{1}\left(\Omega_{(R / I) / k}\right) \leq$ $\mu\left(I+\mathfrak{n}^{3} / \mathfrak{n}^{3}\right)<\operatorname{dim} R=\operatorname{edim} A_{p}$. Hence, the result is a consequence of Theorem 2.3.

Suppose that $(A, m)$ is a reduced Noetherian local ring and that the symmetric algebra $S(M)$ is reduced and not torsionfree. Then the 
basic conclusion (if there is no obstruction on the punctured spectrum) is that $\alpha_{1}(M) \geq \operatorname{edim} A$. In Lemma 2.2, we also have seen that the maximal ideal is generated by the entries of the linear part of $M$. It is natural to ask if the maximal ideal $m$ is actually generated by a set of linear entries, one from each of its corresponding linear syzygies. With a slightly stronger hypothesis, we are able to show this. In the following result, we also include a routine generalization of part of $[7,2.6]$ for convenience and completeness.

Theorem 2.6. Let $(A, m, k)$ be a reduced Noetherian local ring of dimension $d$, and let $M$ be a finitely generated $A$-module of rank $e$ satisfying $\ell\left(M_{p}\right)=\mu\left(M_{p}\right)$ for every prime ideal $p \neq m$. Let $\phi_{1}$ be the linear part of $M$ with respect to some minimal presentation matrix. Suppose that $S(M)$ is reduced but not A-torsionfree.

(i) Then $m$ is generated by the entries of the first $d+e$ rows of $\phi_{1}$.

(ii) If $k$ is algebraically closed, a general row of $\phi_{1}$ generates $m$.

Proof. We first show the second statement. Since the symmetric algebra is reduced, but not $A$-torsionfree, we conclude as usual that $m S(M)$ must be a minimal prime. Let $T$ be the $A$-torsion of $S(M)$. Since $T$ is not contained in $m S$, its image is a nonzero homogeneous ideal in the polynomial ring $S(M) \otimes_{A} k$. Choose a closed point $x \in \mathbb{P}^{n-1}=\operatorname{Proj}\left(S(M) \otimes_{A} k\right)$ not lying on the projective algebraic subset defined by the image of $T$. Then if $P$ is the preimage in $S(M)$ of the ideal of $x$, it follows that $T$ is not contained in the prime $(m S(M), P)=Q$. Since $S(M)_{Q}$ is again reduced, we conclude that $m S(M)_{Q}=0$. In particular, $m(S(M) / P)_{(m)}=0$.

Let $\phi$ be a minimal presentation matrix with linear part $\phi_{1}$, let $T=A\left[T_{1}, \ldots, T_{n}\right]$, so that $S(M) \cong T / J$, where $J=\left(\left[T_{1}, \ldots, T_{n}\right] \phi\right)$. Now, after a possible change of coordinates, we may assume that $P=\left(T_{2}-a_{2} T_{1}, \ldots, T_{n}-a_{n} T_{1}\right) S(M)$, where $a_{i} \in A$ are a lifting of the coordinates of $x$. Let $\phi^{\prime}$ be the matrix obtained from $\phi$ by successively performing the elementary row operation of adding to the first row the scalar multiple of the $i$ th row by $a_{i}$, for $i=2, \ldots, n$.

It follows that the ring $S(M) / P \cong A\left[T_{1}\right] / T_{1} K$, where $K$ is the ideal of $A$ generated by the first row of $\phi^{\prime}$. Therefore, it follows that 


$$
\begin{aligned}
0 & =m(S(M) / P)_{(m)}=m A\left[T_{1}\right]_{m A\left[T_{1}\right]} /\left(T_{1} K\right)_{m A\left[T_{1}\right]} \\
& =m A\left[T_{1}\right]_{m A\left[T_{1}\right]} / K_{m A\left[T_{1}\right]},
\end{aligned}
$$

from which we conclude by flatness that $m=K$. If $K_{1}$ denotes the ideal generated by the linear part of $\phi^{\prime}$, then we also have that $m=K \subset K_{1}+m^{2}$; hence, by Nakayama's lemma, $m=K_{1}$. Since the choice of the point $x \in \mathbb{P}^{n-1}$ is general, this is a general row ideal of the linear part of $M$.

Now the second statement follows as in the first part of the proof of $[7,2.6]$, using instead the $(d+e)$-dimensional prime $P=$ $\left(m S(M), T_{d+e+1}, \ldots, T_{n}\right) S(M)$. One uses the basic fact that $\ell(M) \leq$ $d+e-1([\mathbf{8}, 2.3])$.

Example 2.7. Let $(A, m)$ be a reduced Noetherian local ring with residue field $k$. Then $S(k)$ is reduced, but not $A$-torsionfree, as long as $A \neq k$. In this case, $\beta_{1}(k)=\alpha_{1}(k)=\operatorname{edim} A$ and $m$ is generated by the (sole) row of any minimal presentation matrix of $k$.

Remark 2.8. In $[7,2.6]$, it is shown that a one-dimensional complete local domain $A$, with algebraically closed residue field $k \subset A$, can have $S\left(\Omega_{A / k}\right)$ reduced only if $A$ is regular. (Here $\Omega_{A / k}$ is the universally finite module of differentials.) Since in dimension 1 , only a free module can be of linear type (for modules with a rank), by Theorem 2.6, this will follow once one shows that the maximal ideal cannot be generated by every two ( $e=d=1$ in this case) rows of a (transposed) Jacobian matrix. They verify this, under the additional hypotheses; this part is more elementary. Interestingly, we cannot deduce this theorem from using statement (ii) of the theorem; in this situation, the general row can in fact generate $m$.

3. Normal symmetric algebras. In this section, we consider the case that the symmetric algebra is normal. Again, there must be sufficiently many linear relations on the module, the exact bound now depending on the number of generators and the embedding codimension of $A$. 
Proposition 3.1. Let $(A, m)$ be a Noetherian local ring, and let $M$ be a finitely generated generically free A-module with minranke. Suppose that $S(M)$ is equidimensional and $S(M)_{m S(M)}$ is regular. Then $\alpha_{1}(M) \geq \operatorname{ecodim} A+\mu(M)-e$.

Proof. As usual, let $\phi$ be a minimal presentation matrix with $n$ rows, with linear part $\phi_{1}$ having $r$ columns, set $T=A\left[T_{1}, \ldots, T_{n}\right]$, let $L=\left(\left[T_{1}, \ldots, T_{n}\right] \phi\right)$ and $L_{1}=\left(\left[T_{1}, \ldots, T_{n}\right] \phi_{1}\right)$. Then $S(M)_{m S(M)} \cong$ $(T / J)_{m T}$ is regular. Let $\rho$ be its dimension. Let $x_{1}, \ldots, x_{\rho}$ be elements of $m T$ whose images form a regular system of parameters. Then

$$
m T_{m T}=J_{m T}+\left(x_{1}, \ldots, x_{\rho}\right) T_{m T} \subset\left(L_{1}, x_{1}, \ldots, x_{\rho}\right) T_{m T}+m^{2} T_{m T} ;
$$

hence, $m T_{m T}=\left(L_{1}, x_{1}, \ldots, x_{\rho}\right) T_{m T}$ by Nakayama's lemma. Hence,

$$
\operatorname{edim} A=\mu(m)=\mu\left(m T_{m T}\right) \leq \mu\left(L_{1} T_{m T}\right)+\rho \leq r+\rho .
$$

It follows that $\alpha_{1}(M) \geq \operatorname{edim} A-\rho$.

It remains to identify $\rho$. Since the symmetric algebra is equidimensional its dimension formula $([\mathbf{3}, \mathbf{9}])$ implies that $\operatorname{dim} S(M)=\operatorname{dim} A+e$. Therefore,

$$
\begin{aligned}
\rho & =\operatorname{dim} S(M)_{m S(M)} \leq \operatorname{dim} S(M)-\operatorname{dim} S(M) / m S(M) \\
& =\operatorname{dim} A+e-\mu(M) .
\end{aligned}
$$

(Equality holds if $A$ is universally catenary.) Hence, the result follows.

The next result now follows from Proposition 3.1 as in $[7,2.10]$. We give a proof for completeness.

Theorem 3.2. Let $A$ be a universally catenary normal domain, and let $M$ be a finitely generated $A$-module of rank e satisfying

$$
\alpha_{1}\left(M_{p}\right)<\operatorname{ecodim} A_{p}+\mu\left(M_{p}\right)-e
$$

for every $p \in \operatorname{Spec} A$ with $\mu\left(M_{p}\right)=\operatorname{dim} A_{p}+e-1>e$.

If $S(M)$ is normal, then $S_{i}(M)$ is reflexive for every $i \geq 1$.

Proof. Since $S(M)$ is normal, it suffices to check the equality of the algebras $S(M) \subset \oplus_{i \geq 0} S_{i}(M)^{* *}$ locally at the height one primes of the symmetric algebra. Localizing at such a prime $P$, and at its contraction 
to $A$, changing notation, we may assume that the prime contracts to the maximal ideal $m$ of a local $\operatorname{ring} A$. If $\operatorname{dim} A=0$, then $M$ is free and the result is clear. If $\operatorname{dim} A>0$, then $P=m S(M)$, so $S(M)_{m S(M)}$ is regular. Therefore, by Proposition 3.1, $\alpha_{1}(M) \geq \operatorname{ecodim} A+\mu(M)-e$. But

$$
1=\operatorname{ht} P=\operatorname{dim} S(M)-\operatorname{dim} S(M) / P=\operatorname{dim} A+e-\mu(M) .
$$

By our hypothesis, we conclude that $\mu(M)=e$. Hence, $M$ is free and again the equality of algebras follows.

The last two results recover [7, 2.9 (a), 2.10 (i)], as applied to the module of differentials. We refer the reader to this paper for the corresponding statements, as well as some sharper statements in characteristic zero.

Corollary 3.3. Let $A$ be a universally catenary normal domain, and let $M$ be a finitely generated $A$-module of rank e that is free locally in codimension 2 and satisfies $\mu\left(M_{p}\right) \leq \operatorname{dim} A_{p}+e-2$ for all primes $p \neq m$ with $\operatorname{dim} A_{p} \geq 3$.

If $\alpha_{1}(M)<\operatorname{ecodim} A+\mu(M)-e$ and $S(M)$ is normal, then $S_{i}(M)$ is reflexive for every $i \geq 1$.

4. Ideal case. We now wish to apply the results to the ideal case. In particular, we would like to consider the situation for the conormal module $I / I^{2}$ of an ideal $I$. This closely parallels [7] again; as for $A$ essentially of finite type over a field $k$, one has $\Omega_{A / k} \cong \mathbb{D} / \mathbb{D}^{2}$, where $\mathbb{D}$ is the diagonal ideal. However, here we would like to focus on the case of ideals in a regular ambient ring. This will allow us to use [4] to obtain a slightly stronger result than would follow by applying the theorem directly. This result of [4] asserts that, for a prime ideal $I$ in a regular local ring, the reducedness of the associated graded ring $g r_{I}(R)$ always implies its integrality. In the latter case, we say that the prime ideal is normally torsionfree; this is equivalent to the equality $I^{(n)}=I^{(n)}$ between the powers and symbolic powers, for all $n$.

We shall focus on the question of reducedness. (For the conormal module in a regular ambient ring, the normality of its symmetric algebra is a strong enough condition by itself, roughly like the situation [4].) We would like to give some examples showing the sharpness of 
the result in this case. The work [7] already clearly demonstrated the sharpness in the case for the module of differentials, but here again we would like to give examples of ideals in a regular ring that shows the optimality.

One slightly different form of the main result for the conormal module is as follows.

Theorem 4.1. Let $(R, m)$ be a regular local ring, and let $I$ be a prime ideal that is of linear type locally on the punctured spectrum. Suppose that $\beta_{1}(I)<\operatorname{dim} R$. If $S_{R / I}\left(I / I^{2}\right)$ is reduced, then $I$ is normally torsionfree and of linear type.

Proof. We first note that the conclusion is equivalent to the integrality of $S_{R / I}\left(I / I^{2}\right)$. Indeed, with the hypothesis on $I$, factoring out the $A$-torsion factors through the natural map $S_{R / I}\left(I / I^{2}\right) \rightarrow \operatorname{gr}_{I}(R)$, which, in general, is an isomorphism precisely when $I$ is of linear type [9]. Since a presentation matrix of the conormal module is obtained by base change from one for $I$, this will follow from Theorem 2.3 once we show that the conormal is of linear type on the punctured spectrum, i.e., that the conclusion holds for $I$ locally on the punctured spectrum. But, at such a localization, by assumption, the symmetric algebra is isomorphic to the associated graded ring. Since the former is reduced, so is the latter, and therefore the ideal is normally torsionfree by [4].

In summary, we obtain several constraints for a counterexample for the conormal module.

Corollary 4.2. Let $(R, m)$ be a regular local ring of dimension d, and let $I$ be a prime ideal that is of linear type locally on the punctured spectrum. Suppose that $S_{R / I}\left(I / I^{2}\right)$ is reduced but not integral. Then the following hold:

(i) I is not of linear type;

(ii) $\beta_{1}(I) \geq d$;

(iii) If the fiber ring $\mathcal{R}(I) \otimes_{R} k$ is reduced, then $I$ is normally torsionfree.

Proof. The first statement follows from [4] and the second immediately from Theorem 4.1 . 
For the third, suppose this is not the case. Then, by [4], $G=\operatorname{gr}_{I}(R)$ is not reduced. But, since $\mathcal{R}(I) \otimes_{R} k=\operatorname{dim} G / m G, m G$ is reduced. Thus, $m G$ must intersect the torsion of $G$ nontrivially. But, then $m S_{R / I}\left(I / I^{2}\right)$, being the only other minimal prime, meets the torsion of $S_{R / I}\left(I / I^{2}\right)$ nontrivially, contradicting its reducedness.

We may obtain a class of examples by using the following technical result, which follows from the results of [5].

Remark 4.3. Let $(R, m, k)$ be a regular local ring of dimension $d$ with $k$ infinite, and let $I$ be a prime ideal that is of linear type and normally torsion free, both locally on the punctured spectrum of $R$. Suppose that $I$ has analytic spread $\ell$ and $\mu(I)=\ell+1 \leq d$, that depth $R / I \geq d-\ell$, that $I$ satisfies the Artin-Nagata condition $A N_{\ell-2}$ and that $I_{1}(\phi)=m$ for a minimal presentation matrix $\phi$ of $I$. Then $S_{R}(I)$ and $S_{R / I}\left(I / I^{2}\right)$ are reduced but not integral.

The following are probably the simplest examples of this type.

Example 4.4. Let $I \subset k[x, y, z, w]=R$ be the homogeneous ideal defining the twisted quartic curve $x=s^{4}, y=s^{3} t, z=s t^{3}, w=t^{4}$ in $\mathbb{P}^{3}$. Then, $S_{R}(I)$ and $S_{R / I}\left(I / I^{2}\right)$ are reduced but not integral.

Indeed, in this case, it is well known that $\beta_{1}(I)=\mu(I)=4=\operatorname{dim} R$, and the symmetric algebra of $I$ is reduced but not integral [6].

Example 4.5. Let $I=I_{2}(X) \subset k[X]=R$ where $X$ is a generic $2 \times 4$ matrix over a field $k$. Then $S_{R}(I)$ and $S_{R / I}\left(I / I^{2}\right)$ are reduced but not integral.

It is well known [1] that $I$ is a perfect ideal of codimension 3, is not of linear type, but, having a linear presentation, $\mu(I)=6$ and $\beta_{1}(I)=8=\operatorname{dim} R$.

In both examples, the ideal is homogeneous, so the condition on the reducedness of the fiber cone of Corollary 4.2 (iv) holds.

We remark that in Corollary 4.2 (ii) the stronger statement would involve $\alpha_{1}$ rather than simply $\beta_{1}$. Thus, one may obtain further 
examples that are optimal, or close it, by taking into account the nonlinear syzygies of $I$. (See also [5] for some examples, at least after deformation.)

\section{REFERENCES}

1. W. Bruns and U. Vetter, Determinantal rings, Lect. Notes Math. 1327, Springer-Verlag, New York, 1988.

2. D. Eisenbud, C. Huneke and B. Ulrich, What is the Rees algebra of a module?, Proc. Amer. Math. Soc. 131 (2003), 701-708.

3. C. Huneke and M. Rossi, The dimension and components of symmetric algebras, J. Alg. 98 (1986), 200-210.

4. C. Huneke, A. Simis and W.V. Vasconcelos, Reduced normal cones are domains, Contemp. Math. 88 (1989), 95-101.

5. M.R. Johnson, Depth of symmetric algebras of certain ideals, Proc. Amer. Math. Soc. 129 (2000), 1581-1585.

6. M. Morales and A. Simis, Symbolic powers of monomial curves in $\mathbf{P}^{3}$ lying on a quadric surface, Comm. Alg. 20 (1992), 1109-1121.

7. A. Simis, B. Ulrich and W.V. Vasconcelos, Tangent algebras, Trans. Amer. Math. Soc. 364 (2012), 571-594.

8. _ Rees algebras of modules, Proc. Lond. Math. Soc. 87 (2003), 610-646.

9. W.V. Vasconcelos, Arithmetic of blowup algebras, Lond. Math. Soc. Lect. Notes 195, Cambridge University Press, Cambridge, 1994.

Department of Mathematical Sciences, University of Arkansas, FayetTEVILLE, AR 72701

Email address: markj@uark.edu 